\title{
A STOCHASTIC H-U MODEL FOR MALARIA TRANSMISSION VIA MARKOV
}

\section{THEORY}

Arku, Dennis ${ }^{1}$ and Kallah-Dagadu, Gabriel ${ }^{2}$.

${ }^{1,2}$ Department of Statistics and Actuarial Science, School of Physical ad Mathematical Science, University of Ghana, Legon.

Corresponding Author: Dennis Arku, denarku.gh@gmail.com.

Co-Author: Gabriel Kallah-Dagadu, gkdagadu33@gmail.com

\begin{abstract}
The purpose of this study is to estimate the mean transitioning probabilities from a Healthy state to malaria positive uncomplicated state or to malaria positive severe state. It also classifies the various transitioning probabilities of moving through the various states based on some baseline characteristics. Malaria test results for 2019 over a 12-month period were collected from the University of Ghana school clinic. An H-U model for the study was developed and the transition rates from the cross-sectional data are indicated. With two states Healthy $(\mathrm{H})$ and Uncomplicated (U) forming a state space, there were four possible transitions. The results show that the probability of transitioning from a Healthy state to a malaria positive state is $0.03 \%$ while the probability that an individual will remain at Healthy state $(\mathrm{H})$ after the test is $99.73 \%$. It was found that if an individual is already positive and has taken medication the probability that its second test came out negative is $6.45 \%$ while the chances that it will remain positive but uncomplicated is $93.55 \%$. The study also showed that in the long run, about $95.98 \%$ of persons who visited the student clinic with malaria symptoms recorded negative tests for malaria parasite while about $4 \%$ recorded positive for malaria. In terms of disaggregation by gender, it was realized that the number of reported negative test results were higher for females (97.08\%) than for males $(96.13 \%)$. However,
\end{abstract}


the infection rate is higher for males $(3.87 \%)$ than females $(2.92 \%)$. It is recommended that in as much as the University of Ghana has two health centers (a clinic and hospital), there should be a centralized system to track students' health so research done would not be biased.

Keywords: malaria model, transition matrix, Markov chain, malaria statistics

\section{INTRODUCTION}

Malaria is a life-threatening disease caused by parasites that are transmitted to people through the bites of infected female Anopheles mosquitoes. According to WHO (2017) estimate, there were about 219 million cases of Malaria in 87 countries with estimated number of 435,000 malaria deaths. It is believed that with first symptoms of fever, headache, and chills sometimes mild or severe, it requires that a test be conducted is conducted to determine the symptoms shown is malaria. If malaria is not treated within 24 hours, P. falciparum malaria can progress to severe illness, often leading to death.

There are two states of malaria: Uncomplicated Malaria and Severe Malaria. It is also known that malaria parasites relapse and according to WHO, effective surveillance is required at all points on the path to malaria elimination. Stronger malaria surveillance systems are urgently needed to enable a timely and effective malaria response in endemic regions, to prevent outbreaks and resurgences, to track progress, and to hold governments and the global malaria community accountable (WHO, 2017).

Many researches have been made about malaria and how to model its transmission mathematically. A study by Nedelman (1985) indicated that mosquitoes transmitted malaria. Nedelman limited his work to infants. He considered other works which expanded on what Roland Ross did. Nedelman also conducted a reanalysis of Ross's model to explain Lotka's interpretation of those models. The study was of the view that malaria models had inoculation rate which was the probability per unit time an uninfected individual becomes infected.

Odongo et al., (2015), also made a research to investigate anti-malarial drug use patterns among women who have recently been infected with malaria before and during pregnancy in Uganda. He used data collected through questionnaires and applying a first order Markov probability model to 
help estimate probabilities of transitioning between treatment choices made and during pregnancy. Odongo et al. (2015), used three transition probability transitioning states for their model. The study made two assumptions when constructing the probability model: a constant time interval for each woman, between malaria infection before and during pregnancy and that each medicine was always considered in its rightful dose and duration. While these assumptions are relevant it is quite complicated in practice.

Richard et al. (1993) came up with a three-state Markov model of Plasmodium falciparum Parasitmia. It considered a non-infected state, the infected state exhibiting no clinical signs and the infected state with clinical signs. Data of children in Burkina Faso was used. The article stated that various authors have established that in order to attribute with minimum error a high temperature to malaria attack, it is necessary that concomitantly the level of parasite blood density be greater than a certain threshold, which varies with age. In their model, they made no allowance for misclassification. They also supposed that the parameters were constant between two survey points. The state classification though valid it is limited to the data considerations and classification requirement.

In this paper, we present a Markovian model for the Malaria disease that distinguishes two hypothesized states based on data consistency and threshold requirement: healthy and uncomplicated state. We demonstrate how the transition rates between these two states can be estimated and apply the model to evaluate data collected from the University of Ghana student Clinic for the year 2019. The overall aim is to determine the average transitioning probability between these two states within the given period.

\section{METHODOLOGY}

The article describes a quantitative study of a stochastic model using a Markov chain. Data were obtained from all students who visited the student clinic and were tested for Malaria from January 2019 to December 2019.

\section{Model Analysis strategy}

The pre-analysis was done by checking for missing data and outliers. The outliers were removed and the section of data that did not fall in the category of the target group were also removed. That 
is all values outside the range were considered outliers. It was found out that none of the individuals fell in the category of severe malaria reducing the states for the study to two states. Therefore, the Markov transition matrix becomes a two state Markov chain.

A description of the states under consideration is given as below:

Healthy state: it comprises of individuals who tested negative to the malaria test and individuals who have recovered from the malaria infection or disease.

Uncomplicated state: it comprises of infected individuals or individuals tested positive with the malaria parasite count of less than 100,000.

Model Development for the two-state chain.

Consider two discrete states: Healthy state (State 0) and Uncomplicated state (State 1). If $\left(X_{h}, h=0,1\right)$ represent the number of individuals at any state for Malaria at any time $t$, then clearly, $X_{i}$ is a stochastic process with states 0 and 1 .

Thus, the first-order time-homogeneous Markov dependency can statistically be modeled as

$$
\begin{gathered}
P\left(X_{n}=h_{n} \mid X_{n-1}=h_{n-h, \ldots,}, X_{1}=h_{1}, X_{n}=X_{0}\right) \\
=P\left(X_{n}=h_{n} \mid X_{n-1}=h_{n-1}\right)
\end{gathered}
$$

Then, the transition probability $\left(P_{h u}\right)$ for $h, u=0,1$ is denoted in matrix form as

$$
\left(P_{h u}\right)=\left(\begin{array}{ll}
P_{00} & P_{01} \\
P_{10} & P_{11}
\end{array}\right)
$$

where

$$
\sum_{u=0}^{1} P_{h u}=1, h=0,1 .
$$

Parameters of the Markov Chain (Probabilities of Transition matrix), Phh: probability of remaining in a state $h$. and $\mathrm{Phu}$ : transition probability from state $h$ to state $u$.

Remarks. The parameter $\mathrm{P}_{01}$ is mostly referred to in the literature as discrete time force of infection. Also, $\mathrm{P}_{10}$ is the recovery or defection probability. The time step unit to ensure the transition from one state to another is measured on a yearly basis. 


\section{Model assumptions}

In relation to the model, the following assumptions are made.

1. The current state of an individual is only dependent on only the individuals state or step or health status according to the malaria test.

2. Transitioning probabilities are independent of time and remain constant over the study period.

3. Successive transitions or relapse confirmed severity of the disease and other medical complications were not taken into consideration.

4. Students who do not return to the clinic after treatment were considered healed.

\section{Estimating Transition Probabilities.}

The maximum- likelihood estimation (MLE) was used to estimate the transition probabilities for the Malaria disease with its standard errors. Table 1 shows the number of individuals during the study period at any state $(\mathrm{H}, \mathrm{U})$ for each case study of the Malaria disease.

Table 1: Number of individuals at any of the two state for group

\begin{tabular}{lcc}
\hline Group & Healthy & Uncomplicated \\
\hline Healthy Individual & $\mathrm{X}_{00}$ & $\mathrm{X}_{01}$ \\
Uncomplicated & $\mathrm{X}_{10}$ & $\mathrm{X}_{11}$ \\
individual & & \\
\hline
\end{tabular}

$\mathrm{X}_{00}$ : number of healthy individuals who remained healthy at the end of the study period.

$\mathrm{X}_{01}$ : number of healthy individuals who became infected and at the uncomplicated stage at the end of the study period.

X10: number of infected individuals who recovered at the end of the study period.

X11: number of uncomplicated individuals who remained at the uncomplicated stage at the end of the study period.

The transition events are independent of one another (as defined by the Markov principle); the likelihood of the transition probability, $\mathrm{Phu}$, follows a binomial model: 


$$
L\left(P_{h u} \mid N, x\right)=\left(\begin{array}{c}
N_{h} \\
X_{h u}
\end{array}\right) P_{h u}^{x_{h u}}\left(1-P_{h u}\right)^{N_{h}-x_{h u}},
$$

Where $N_{h u}$ is the number of observed transition that starts from state $\mathrm{h}$ to $\mathrm{u}$ and

$$
\sum_{u} P_{h u}=1
$$

From equation (4) and the assumption of constant transition probabilities over the period, the transition probability matrix is estimated as a multinomial distribution given as

$$
\widehat{P}=\frac{x_{h u}}{\sum_{u} x_{h u}}=\frac{x_{h u}}{N_{h}}
$$

for $\mathrm{h}, \mathrm{u}=0,1$ with standard errors from the sampling distribution of the ML estimate given as

$$
\hat{S} . e\left(P_{h u}\right)=\sqrt{\frac{\hat{P}_{h u}\left(1-\hat{P}_{h u}\right)}{N_{h}}} \text {. }
$$

\section{Estimating the Malaria Disease Metrics.}

The probability that a Healthy individual becomes infected with malaria for the first time between $\mathrm{m}-1$ and $\mathrm{m}$ time steps for states $\mathrm{h}, \mathrm{u}=0,1$ from the transition probability matrix $(\mathrm{H}-\mathrm{U})$ is given as shown in Table 1.

$$
f_{01}^{(m)}=\left(X_{n+m}=1, X_{n+m-1}=0, \ldots, X_{n+1}=0 \mid X_{n}=0\right)=P_{00}^{m-1} P_{01}
$$

Similarly, the probability that an uncomplicated individual first recovers between $m-1$ and $m$ time steps is given as

$$
f_{10}^{(m)}=\left(X_{n+m}=0, X_{n+m-1}=1, \ldots, X_{n+1}=1 \mid X_{n}=1\right)=P_{11}^{m-1} P_{10}
$$


The expected time of infection to an uncomplicated stage and recovery has a closed form solution which is computed as

$$
E\left(\tau_{h u}^{1}\right)=\frac{\sum_{m=1}^{\infty} m f_{h u}^{m}}{P_{r}(h \rightarrow u)}=\frac{P_{h u}}{1-P_{h h}}
$$

for $\mathrm{h}, \mathrm{u}=0,1, \mathrm{~h} \neq \mathrm{u}$, where the numerator, $\sum f m_{h u}^{m}$, is the expected value of first passage time from state $h$ to state $u$ and the denominator

$$
P_{r}(h \rightarrow u)=\frac{P_{h u}}{1-P_{h h}}
$$

The life expectancies $\left(\mathrm{W}_{\mathrm{h}}, \mathrm{h}=0,1\right)$ for healthy and Uncomplicated individuals can also be estimated using the following equation:

$$
\mathrm{W}=(I-Q)^{-1} \times\left(\begin{array}{l}
1 \\
1
\end{array}\right)
$$

where $\mathrm{I}$ is a $2 \times 2$ identity matrix and $\mathrm{Q}$ is a transition matrix given as

$$
Q=\left(\begin{array}{ll}
P_{00} & P_{01} \\
P_{10} & P_{11}
\end{array}\right)
$$

The $\mathrm{n}^{\text {th }}$ step transition probability matrix $\left(P^{n} h u, \mathrm{~h}, \mathrm{u}=0,1\right)$ was estimated for each disease using a decomposition method that requires eigenvalues and their corresponding eigenvectors. Hence, it can be estimated using the decomposition below:

$$
P^{n}=Q \wedge^{n} Q^{-1}
$$

where $\mathrm{Q}$ is $2 \times 2$ nonsingular matrix and $X_{u},(u=0,1)$ is the right eigenvectors corresponding to the eigenvalues $\lambda_{\mathrm{u}}(\mathrm{u}=0,1)$. Thus,

$$
\begin{array}{r}
P X_{u}=\lambda_{u} X_{u}, \\
\lambda^{n}=\left(\begin{array}{cc}
\lambda_{0}^{n} & 0 \\
0 & \lambda_{1}^{n}
\end{array}\right)
\end{array}
$$


To estimate the limiting distribution we note that,

$$
\begin{aligned}
& \lim _{n \rightarrow \infty} P^{n}=\pi \\
& \pi P=\pi
\end{aligned}
$$

where, $P=\left[\begin{array}{cc}1-a & a \\ b & 1-b\end{array}\right]$ and $\pi=\left(\overline{\pi_{0}}, \overline{\pi_{1}}\right)$, where

\section{RESULTS}

Considering that the H-U model for malaria disease, thus Healthy (State 0) and Uncomplicated (State 1).

Assume $\left(X_{i, i=0,1}\right)$ represent the number of individuals at any state of the Malaria disease at any time $t$, which satisfies the first order time-homogenous Markov dependency from equation (1). Clearly, $\mathrm{X}_{\mathrm{i}}$ satisfies the Markov chain model with a state space $S=\{0,1\}$.

The Table 2-4 show the number of individuals included in the study population from Healthy state to Uncomplicated state for the total Malaria cases and the disaggregated, Female and Males respectively.

\section{Estimating the Transition Probabilities}

A two state Markov chain has the nature where it is seen as 'sticky', where if an individual is healthy at one instance (one step) and in the next instance or step, the person is likely to still be healthy. A manual computation was used in estimating the transition probabilities and for generating the probability transition matrix for each state of the Malaria in all classes. Considering that the Markov chain is in state 0 with a probability of 0.9973 of being healthy, then there would be a 0.0027 probability of moving to state 1 the probability of being in an uncomplicated state.

The transition probability matrices for the various classes are, respectively, presented as follows:

$$
P_{T}=\left[\begin{array}{ll}
0.9973 & 0.0027 \\
0.0645 & 0.9355
\end{array}\right]
$$


where $P_{T}$ is the transition matrix for total malaria cases, and for female and males are given as,

$$
\begin{aligned}
& P_{F}=\left[\begin{array}{ll}
0.9965 & 0.0035 \\
0.1165 & 0.8833
\end{array}\right] \\
& P_{M}=\left[\begin{array}{ll}
0.9984 & 0.0016 \\
0.0397 & 0.9603
\end{array}\right]
\end{aligned}
$$

For the total transition matrix, it is seen that the probability of healthy individuals who became infected and at the uncomplicated stage at the end of the study period is seen to be 0.0027 and the probability of infected individuals who recovered at the end of the study period were 0.645; which shows that the rate of infection of Malaria is generally low and the rate of recovery from Malaria is generally high. It is observed that the probability of healthy individuals who remained healthy at the end of the study period for females is 0.9965 and 0.9984 for males; which shows that most of the males remained healthy than the females. Also the probability of healthy individuals who became infected and at the uncomplicated stage at the end of the study period is seen to be 0.0035 and 0.0016 for females and males respectively; this means that more females got infected by the end of the period compared to males. Again, the probability of infected individuals who recovered at the end of the study period were 0.1165 and 0.0397 for females and males respectively which implies that females recover from Malaria faster than males. Finally, it is found that the probability of uncomplicated individuals who remained at the uncomplicated stage at the end of the study period were 0.8833 and 0.9603 for males and females respectively and this means that males stay infected for a longer period compared to females.

Table 2: Number of individuals at any state for total Malaria cases at the end of the period.

\begin{tabular}{lll}
\hline Group & Healthy State & Uncomplicated State \\
\hline Healthy State & 2923 & 8 \\
Uncomplicated State & 12 & 174 \\
\hline
\end{tabular}


Table 3: Number of individuals at any state for Females in the sample population at the end of the period

\begin{tabular}{lll}
\hline Group & Healthy State & Uncomplicated State \\
\hline Healthy State & 1688 & 6 \\
Uncomplicated State & 7 & 53 \\
\hline
\end{tabular}

Table 4: Number of individuals at any state for Males in the sample population at the end of the period.

\begin{tabular}{lll}
\hline Group & Healthy State & Uncomplicated State \\
\hline Healthy State & 1235 & 2 \\
Uncomplicated State & 5 & 121 \\
\hline
\end{tabular}

\section{Classification of States of the Model using Graph Algorithm}

A graph algorithm was used to represent the transition probabilities for each category of the Malaria disease so as to easily classify the states into recurrent, transient, or absorbing. From Figure $1 \mathrm{~A}$, it can be concluded that it is recurrent, which means that the state communicates between themselves. Figure $1 \mathrm{~B}$ and $\mathrm{C}$ are also seen to be recurrent. It is seen that all states communicate $\mathrm{H} \leftrightarrow \mathrm{U}$ making it an irreducible Markov chain. It is recurrent because starting from a healthy state $\mathrm{H}$, one would eventually return to $\mathrm{H}$ in a finite number of steps, which means the eventual return is certain.

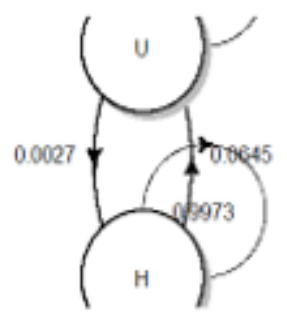

A

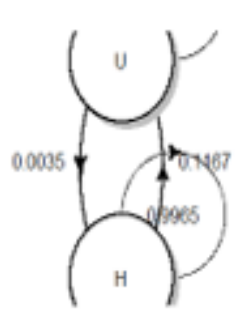

B

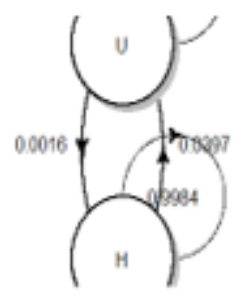

C 
Figure 1: Graphical display of the transition matrices for the three categories; Total(A) malaria cases, Female (B) and Male (C), respectively.

Considering the two main types of recurrent states, Figures 1 can be classified as positive recurrent states. Since all states belong to the same equivalence class, it can be classified as an irreducible Markov chain hence it can be inferred from the classifications that the above Markov chain is ergodic. Since it is also classified as a finite Markov chain. Hence it is clear that $P_{T,} P_{F}$, and $P_{M}$ have limiting distributions. That is $\lim _{n \rightarrow \infty} P^{n}=\pi$. It is seen that $P_{T} P_{F}$, and $P_{M}$ are all square matrices $(2 \times 2)$ which satisfies a stochastic or transition matrix. This is because $\sum_{h \in S} P_{h u}=1$ and for each $\mathrm{u} \in \mathrm{S}, \mathrm{P}_{\mathrm{T}}, \mathrm{P}_{\mathrm{F}}$, and $\mathrm{P}_{\mathrm{M}}$ can be considered as doubly stochastic matrices.

\section{Estimation of the $n$ step transition probability}

Considering a two-state Markov chain transition probability matrix $\mathrm{P}$, then the $\mathrm{n}$-step probability matrix would be $P^{n}$. The $P^{n}$ transition probability matrix was estimated from equation (14). The Markov chains above are all aperiodic and irreducible. The $P^{n}$ transition probability matrix predicts the transition probabilities for each state at any time step.

$$
\begin{aligned}
& P_{T}^{n}=\left[\begin{array}{ll}
\frac{215}{224}+\frac{9(0.9328)^{n}}{224} & \frac{9}{224}-\frac{9(0.9328)^{n}}{224} \\
\frac{215}{224}-\frac{215(0.9328)^{n}}{224} & \frac{215}{224}+\frac{215(0.9328)^{n}}{224}
\end{array}\right] \\
& P_{F}^{n}=\left[\begin{array}{ll}
\frac{233}{240}+\frac{7(0.8800)^{n}}{240} & \frac{7}{240}-\frac{7(0.8800)^{n}}{240} \\
\frac{233}{240}-\frac{233(0.8800)^{n}}{240} & \frac{233}{240}+\frac{233(0.8800)^{n}}{240}
\end{array}\right] \\
& P_{M}^{n}=\left[\begin{array}{ll}
\frac{397}{413}+\frac{16(0.9587)^{n}}{413} & \frac{16}{413}-\frac{16(0.9587)^{n}}{413} \\
\frac{397}{413}-\frac{397(0.9587)^{n}}{413} & \frac{397}{413}+\frac{397(0.9587)^{n}}{413}
\end{array}\right]
\end{aligned}
$$

Clearly, $\mathrm{n}=1$ from the estimated $P^{n}$ transition matrix gives the actual first transition matrix of $P_{T}, P_{F}$, and $P_{M}$, respectively. Therefore, the transition matrix at any time step greater than or equal to 1 can be generated from the fitted $P^{n}$ matrix. 
Estimating steady state or limiting distribution $\left(\lim _{n \rightarrow \infty} P^{n}=\pi\right.$.)

From Equation (14), we can also deduce the limiting distribution for the various classes of the Malaria disease. After the computation, the limiting distributions using equation (19) for $\mathrm{P}_{\mathrm{G}}, \mathrm{P}_{\mathrm{F}}$, and $\mathrm{P}_{\mathrm{M}}$ their solutions are presented below respectively.

$$
\begin{aligned}
& \pi_{T}=(0.9598,0.0402) \\
& \pi_{F}=(0.9708,0.0292) \\
& \pi_{M}=(0.9613,0.0387)
\end{aligned}
$$

Considering the above probabilities, the probability of one being healthy in the long run is almost assured. Hence this proves the temporal immunity one gets after being infected with malaria severally.

\section{DISCUSSION}

According to Sonnenberg et al. (1993), Markov models are useful when a decision problem involves risk that is continuous over time, when the timing of events is important. The study utilized these ideas to be able to arrive at the conclusion. In this study Markov chain model showed that both rate of infection and severity were relatively low in all the three classifications. The findings relate with Twumasi et al. (2019) where similar outcome, was obtained but with different disease scope namely, HIV, Hepatitis B (HBV) and tuberculosis TB). These diseases are classified as infectious diseases. Considering HIV, HBV and TB have different states in terms of treatment and disease progression hence making it possible for it to be modelled using Markov chain modelling. Thus, male had higher chances of staying in the uncomplicated state for a longer period than the females. Also, females had a higher recovery rate than males.

Generally, the probability of an individual recovering from Malaria is high compared to the probability of that person getting infected. Considering the probabilities computed in Equations 4 and 5, the probability of one being healthy in the long run is almost assured. This demonstrative effectiveness in the treatment of malaria. It also proves the temporal immunity one gets after being infected with Malaria severally this support effort of. Sandip, Ram and Somdatta (2011). Sandip et al. (2011) published a review on mathematical models 
of malaria to help public health get a better appreciation of strategies to effectively control malaria.

The study could not extend the state transition matrix to Severe cases due to data unavailability. That notwithstanding, the data and analysis presented is a fair representation of the situation pertaining to the student clinic at University of Ghana.

\section{CONCLUSION}

In conclusion, the Markov chain model has helped to show the transition probabilities between healthy individuals and malaria positive but uncomplicated individuals within the given year. With two states Healthy $(\mathrm{H})$ and Uncomplicated (U) forming a state space, there are four possible transitions. If we are at $\mathrm{H}$ we could transition to $\mathrm{U}$ or stay at $\mathrm{H}$. if we are at $\mathrm{U}$, we can transition to $\mathrm{H}$ or stay at $\mathrm{H}$. Based on the formulated transition probability matrices, the probability of transitioning from Healthy state to malaria positive state is about $0.03 \%$ while the probability that the individual will remain at $\mathrm{H}$ after test is 99.73\%. This means that probability of individuals with malaria symptoms who were eventually tested positive were $0.03 \%$ at first visit. If the individual is already positive and has taken medication the probability that its second test came out negative is $6.45 \%$ while the chances that it will remain positive but uncomplicated is $93.55 \%$.

The studied showed that in the long run about $95.98 \%$ who showed at the student clinic with malaria symptoms recorded negative for malaria parasite while about $4 \%$ record positive for malaria. In terms of disaggregation by gender, it was realized that number of reported negative test results were higher for females $(97.08 \%)$ than for males $(96.13 \%)$. However, the infection rate is higher for males $(3.87 \%)$ than females $(2.92 \%)$.

It is recommended that, in as much as the University of Ghana has two health centers (a clinic and hospital), there should be a centralized system to track students' health for effective and efficient research.

\section{DECLARATION}

Ethical considerations 
The study did not involve physical human contact. However, prior permission was secured from UG hospital administration.

\section{Funding}

None

\section{Competing Interests}

No potential conflict of interest was reported by the authors.

\section{Author contributions}

The first author designed the study and carried out the research work. Both authors carried out the analyses and interpretation of the data. All authors read and approved the final draft of the manuscript.

\section{Acknowledgement}

The authors wish to thank management of University of Ghana Hospital, Legon for making the data available upon request.

\section{Availability of data}

Data is available upon request to the corresponding author.

\section{REFERENCES}

CDC. (2019, October 15). Centers for disease Central and Prevention. Retrieved from Centers for disease Central and Prevention: https://www.cdc.gov/malaria/about/disease.html

Goodman, L. A. (2014). Statistical Inference About Markov Chains. August. https://doi.org/10.1214/aoms/1177707039

Odongo, C. O., Bisaso, K. R., Kitutu, F., Obua, C., \& Byamugisha, J. (2015). Is there a distinction between malaria treatment and intermittent preventive treatment? Insights from a crosssectional study of anti-malarial drug use among Ugandan pregnant women. Malaria Journal, 14(1), 1-9. https://doi.org/10.1186/s12936-015-0702-7

Nedelman, J. (1985). Introductory review some new thoughts about some old malaria models. 
Mathematical Biosciences, 73(2), 159-182. https://doi.org/10.1016/0025-5564(85)90010-0

Richard, A., Richardson, S., \& Maccario, J. (1993). A three-state Markov model of Plasmodium falciparum parasitemia. Mathematical Biosciences, 117(1-2), 283-300. https://doi.org/10.1016/0025-5564(93)90029-A.

Sandip, M, Ram, R. S. \& Somdatata, S. (2011). Mathematical modelling of malaria-a review. 10(202),retrieved from https://malariajournal.biomedcentral.com/articles/10.1186/14752875-10-202.

SevereMalariaObservatory. (2019, October 28). Severe Malaria Observatory. Retrieved from Severe Malaria Observatory website: https://www.severemalaria.org/countries/ghana

Sonnenberg, F. A., Beck, J. R., Frank, A., \& Beck, J. R. (1993). Markov Models in Medical Decision Making : A Practical Guide. https://doi.org/10.1177/0272989X9301300409

Twumasi, C., Asiedu, L., \& Nortey, E. N. N. (2019). Markov Chain Modeling of HIV, Tuberculosis, and Hepatitis B Transmission in Ghana. Interdisciplinary Perspectives on Infectious Diseases, 2019, 1-8. https://doi.org/10.1155/2019/9362492

WHO. (2019, October 11). World health organization. Retrieved from world health organization website: https://www.who.int/malaria/areas/treatment/en/

WHO. (2019, October 11). World health organization. Retrieved from world health organization website: https://www.who.int/news-room/fact-sheets/detail/malaria 